\title{
Comment on "Spin and statistics in nonrelativistic quantum mechanics: The spin-zero case"
}

\author{
R. E. Allen and A. R. Mondragon \\ Center for Theoretical Physics, Texas A\&M University, College Station, Texas 77843, USA
}

(Received 11 April 2003; published 14 October 2003)

\begin{abstract}
We emphasize that there is no spin-statistics connection in nonrelativistic quantum mechanics. In several recent papers [including Phys. Rev. A 67, 042102 (2003)], quantum mechanics is modified so as to force a spin-statistics connection, but the resulting theory is quite different from standard physics.
\end{abstract}

DOI: 10.1103/PhysRevA.68.046101

PACS number(s): 03.65.Ta

It has been known for many years that there is a spinstatistics connection in relativistic quantum field theory [1-4] but not in nonrelativistic quantum mechanics [5]. However, several recent papers [6-8] have led to some confusion regarding the second point.

Let us first remind ourselves why there is no spinstatistics theorem in nonrelativistic quantum mechanics. The essential reason is that the restrictions that imply a spinstatistics connection in relativistic field theory are no longer meaningful in nonrelativistic physics. For example, Weinberg's textbook [3] provides a relatively simple and physical proof based on microcausality, or the requirement that commutators associated with observable quantities vanish for spacelike separations. In nonrelativistic physics, causality is still a meaningful requirement, but microcausality is not, because there is no longer a light cone. This proof then does not apply in the nonrelativistic case, and the same is true of the other proofs based on Lorentz invariance.

There are nonrelativistic wave functions for either $N$ fermions or $N$ bosons with any spin $(0,1 / 2,1,3 / 2, \ldots)$. For example, a basis function with the form

$$
\Psi\left(\mathbf{r}_{1}, \mathbf{r}_{2}\right)=\left(\phi_{1}\left(\mathbf{r}_{1}\right) \phi_{2}\left(\mathbf{r}_{2}\right)-\phi_{1}\left(\mathbf{r}_{2}\right) \phi_{2}\left(\mathbf{r}_{1}\right)\right) / \sqrt{2}
$$

is acceptable for spin-zero fermions, where $\phi$ is a simple scalar. More generally, a basis function with the form

$$
\begin{aligned}
\Psi\left(\mathbf{r}_{1}, \mathbf{r}_{2}, \ldots, \mathbf{r}_{N}\right) & =\mathcal{A} \prod_{i=1}^{N} \psi_{i}\left(\mathbf{r}_{i}\right), \quad \text { fermions } \\
& =\mathcal{S} \prod_{i=1}^{N} \psi_{i}\left(\mathbf{r}_{i}\right), \quad \text { bosons }
\end{aligned}
$$

is appropriate for $N$ particles with any spin. Here $\mathcal{A}$ or $\mathcal{S}$ represents antisymmetrization or symmetrization of the product (with insertion of the correct normalization factor). Each $\psi$ is a function corresponding to the desired spin $s$; for example, $\psi$ is a two-component spinor if $s=1 / 2$. A nonrelativistic field theory can then be constructed in the usual way, having fermions or bosons with any spin [9]. The field operator consistently transforms as both a field and a quantum operator [5].

According to Ref. [6], on the other hand, Eq. (1) is not an acceptable wave function. This conclusion was reached because quantum physics was modified by adding an unusual constraint: In the words of Ref. [6], "The approach used here is based on the requirement that the point $\left\{\mathbf{r}_{1}, \mathbf{r}_{2}\right\}$ in the configuration space for two identical spinless particles is the same point as $\left\{\mathbf{r}_{2}, \mathbf{r}_{1}\right\}$." But this requirement implies that the wave function must return to its original value when $\left(\mathbf{r}_{1}, \mathbf{r}_{2}\right)$ is transformed to $\left(\mathbf{r}_{2}, \mathbf{r}_{1}\right)$ :

$$
\Psi\left(\mathbf{r}_{2}, \mathbf{r}_{1}\right)=\Psi\left(\mathbf{r}_{1}, \mathbf{r}_{2}\right) .
$$

I.e., the two-particle wave function is only allowed to acquire the + sign appropriate for bosons, and is forbidden to acquire the - sign appropriate for fermions. It is this requirement that forbids spin-zero fermions with wave function (1). In Ref. [6], therefore, the spin-statistics connection is simply imposed by fiat.

Essentially the same philosophy was used in Refs. [7] and [8], but generalized to arbitrary spin, so that an interchange of particles requires an interchange of both positions and spins. In the words of Ref. [7], "we must identify the points $\mathbf{r}$ and $-\mathbf{r}$, since these correspond to complete interchange of the particles (positions and spins) and so are indistinguishable." They then conclude that

$$
|\Psi(-\mathbf{r})\rangle=|\Psi(\mathbf{r})\rangle,
$$

where $|\Psi(\mathbf{r})\rangle$ specifies the state of the two particles. Again, in the simplest case $s=0$, fermions have clearly been banished at the outset.

If one does not impose the unusual constraint (4) or (5), nonrelativistic bosons are allowed to have any spin $(0,1 / 2,1,3 / 2, \ldots)$ and the same is true of nonrelativistic fermions.

This work was supported by the Robert A. Welch Foundation. 
[1] W. Pauli, Phys. Rev. 58, 716 (1940).

[2] R.F. Streater and A.S. Wightman, PCT, Spin and Statistics, and All That (Princeton University Press, Princeton, 2000).

[3] S. Weinberg, The Quantum Theory of Fields (Cambridge University Press, Cambridge, 1995), Vol. 1, Chap. 5.

[4] I. Duck and E.C.G. Sudarshan, Pauli and the Spin-Statistics Theorem (World Scientific, Singapore, 1997).

[5] A.S. Wightman, Electron. J. Diff. Eqns., Conf. 04, 2000, pp.
207-213; http://ejde.math.swt.edu

[6] M. Peshkin, Phys. Rev. A 67, 042102 (2003); e-print quant-ph/0207017.

[7] M.V. Berry and J.M. Robbins, Proc. R. Soc. London, Ser. A 453, 1771 (1997).

[8] M.V. Berry and J.M. Robbins, J. Phys. A 33, L207 (2000).

[9] A.L. Fetter and J.D. Walecka, Quantum Theory of ManyParticle Systems (McGraw-Hill, San Francisco, 1971). 